\title{
Baicalin inhibits the expression of monocyte chemoattractant protein-1 and interleukin-6 in the kidneys of apolipoprotein E-knockout mice fed a high cholesterol diet
}

\author{
LIHUA LIU, PINGPING LIAO, BIN WANG, XIN FANG, WEI LI and SIMING GUAN \\ Department of Geriatrics, Union Hospital, Tongji Medical College, Huazhong University of Science and Technology, \\ Wuhan, Hubei 430022, P.R. China
}

Received April 14, 2014; Accepted November 25, 2014

DOI: $10.3892 / \mathrm{mmr} .2015 .3186$

\begin{abstract}
Hyperlipidemia is considered an independent risk factor for renal dysfunction and induces a significant increase in the expression of inflammatory mediators, which can be used to evaluate the degree of renal injury. Baicalin is widely used in traditional Chinese herbal medicine and has multiple pharmacological effects. The present study investigated whether baicalin can attenuate the expression of vascular cell adhesion molecule 1 (VCAM-1) via a reduction in the expression of monocyte chemoattractant protein-1 (MCP-1) and interleukin-6 (IL-6) in the kidney of apolipoprotein E (ApoE)-knockout (KO) mice fed a high cholesterol diet. These mice were used as a model of atherosclerosis and were treated with baicalin $(100 \mathrm{mg} / \mathrm{kg} / \mathrm{day})$ daily by gavage for a period of 12 weeks. By contrast, wild-type male C57BL/6J mice were fed a standard diet. Blood samples were obtained from the angular veins of the mice to measure the total cholesterol (TC) and the expression levels of VCAM-1, MCP-1 and IL- 6 in the kidney tissues of the mice were analyzed using reverse transcription quantitative polymerase chain reaction and western blot analysis. Following oral administration of baicalin, no significant difference was observed in the TC in the baicalin group compared with the high cholesterol diet control group. The TC was significantly higher in the AopE-KO mice compared with the wild-type male C57BL/6J mice. The expression levels of VCAM-1, MCP-1 and IL-6 in the kidney tissues of the baicalin group were lower compared with those in the high cholesterol diet control group. The results suggested that baicalin decreased the expression levels of pro-inflammatory mediators and prevented kidney dysfunction in the ApoE-KO mice fed a high cholesterol diet.
\end{abstract}

Correspondence to: Dr Siming Guan, Department of Geriatrics, Union Hospital, Tongji Medical College, Huazhong University of Science and Technology, 1277 Jiefang Avenue, Wuhan, Hubei 430022, P.R. China

E-mail: smguan16@163.com

Key words: baicalin, monocyte chemoattractant protein-1, interleukin-6, vascular cell adhesion molecule 1

\section{Introduction}

Lipid accumulation in the kidney contributes to the progression of chronic kidney disease and independently induces renal dysfunction $(1,2)$. Hyperlipidemia induces a significant increase in the expression of monocyte chemoattractant protein-1 (MCP-1) in apolipoprotein E (ApoE)-/- mice fed a high-fat $\operatorname{diet}(3,4)$.

During the process of kidney injury, the expression of MCP-1 is significantly increased via numerous cells expressing MCP-1 in kidney inflammation, including those in glomerular (5-7) and tubular areas (8-10) and primary endothelial cells (11). Tashiro et al suggested that urinary MCP-1 may be useful for evaluating the degree of renal injuries in patients with diabetic nephropathy (12). By contrast inhibition of the expression of MCP-1 protects renal injury by reducing macrophage infiltration $(13,14)$.

The increased expression of MCP-1 and subsequent macrophage infiltration is a substantial source of pro-inflammatory cytokines, including tumor necrosis factor (TNF)- $\alpha$ and interleukin (IL)-6, which induce further damage in kidney dysfunction. Increased expression levels of IL-6 are often observed in patients with progressive kidney dysfunction and can affect the outcome of long-term kidney diseases (15), suggesting that MCP-1 and IL-6 are important in hyperlipidemia-induced kidney dysfunction.

Baicalin is one of several pharmacologically-active flavones and is widely used in traditional Chinese herbal medicine (16). It has a number of pharmacological effects, including inhibitory effects against several viruses (17-19) and anti-inflammatory, anti-allergic and antibacterial effects in a variety of inflammatory diseases $(20,21)$. However, the effects of baicalin on MCP-1 and IL-6 in the kidneys of ApoE-knockout (KO) mice have not been investigated.

Due to its anti-inflammatory effect, baicalin may have a protective effect against kidney dysfunction via regulating the expression of MCP-1 and IL-6.

\section{Materials and methods}

Drug administration. Baicalin (Sigma-Aldrich, St. Louis, MO, USA; cat. no. 21967-41-9; purity, 98\%; molecular formula, 
$\mathrm{C}_{21} \mathrm{H}_{18} \mathrm{O}_{11}$; molecular weight, 446.36) was dissolved in normal saline and the $\mathrm{pH}$ was adjusted to $\mathrm{pH} 7.4$ using $\mathrm{NaOH}$.

Experimental animal models and tissues preparation. Male C57BL/6J mice, and ApoE-/- mice (8-week-old) which were of a C57BL/6J background, were purchased from The Jackson Laboratory (Bar Harbor, ME, USA). The mice were bred and maintained at the Animal Center of Beijing University (Beijing, China), following the guidelines provided in the Guide for the Care and Use of Laboratory Animals, published by the US National Institutes of Health (NIH Publication No. 85-23, revised 1996). All animal procedures were reviewed and approved by the ethics committee of Huazhong University os Science and Technology (Wuhan, China). The mice were acclimated to their environment, which consisted of controlled lighting (12 h light/dark cycle) and temperature $\left(25^{\circ} \mathrm{C}\right)$, for a period of 1 week.

Following adaptation, the ApoE-/- mice were randomly assigned to one of two groups. Group 1 (hypercholesterol control group; $n=6$ ) were freely fed a high-cholesterol diet containing $1.25 \%$ cholesterol and $10 \%$ coconut oil (22) and received normal saline by gavage feeding once daily for 12 weeks. Group 2 (baicalin administration group; $n=6$ ) were fed a high-cholesterol diet (100 mg/kg/day) and received baicalin treatment $(200 \mu \mathrm{l} / \mathrm{kg} /$ day $)$ by gavage feeding once daily for 12 weeks. A group of wild-type male C57BL/6J mice (normal control group, $n=6$ ) were fed a standard diet (free feeding; mouse food, Oriental Yeast, Co, Ltd., Tokyo, Japan) and received normal saline by gavage feeding once daily for 12 weeks. Water was available ad libitum to all mice groups.

At 21 weeks of age, the mice were anesthetized with $2 \%$ pentobarbital solution (Wuhan Boster Biological Technology, Ltd., Wuhan, China) by intraperitoneal injection, and then sacrificed by bleeding through the aorta. The heart and aorta were immediately removed following perfusion with phosphate-buffered saline (PBS; Wuhan Boster Biological Technology, Ltd., Wuhan, China). The kidneys of the mice were then rapidly frozen in liquid nitrogen (Huazhong University of Science and Technology, Wuhan, China) and stored until subsequent protein and RNA extraction.

Plasma TC profile. The mice were anesthetized at 21 weeks of age and blood was obtained from the angular veins using a capillary siphon. The plasma levels of TC were detected using a Hitachi 917 auto-analyzer (Hitachi, Ltd., Tokyo, Japan).

Reverse transcription quantitative polymerase chain reaction $(R T-q P C R)$ analysis of the mRNA expression levels of MCP-1,IL-6 and vascular cell adhesion molecule 1 (VCAM-1). Total RNA was extracted from the mouse renal tissues using TRIzol reagent (Invitrogen Life Technologies, Carlsbad, CA, USA) according to the manufacturer's instructions. mRNA was then converted into cDNA with the ReverTra Ace ${ }^{\circledR}$ qPCR RT Kit (Toyobo, Osaka, Japan). Following amplification by RT, the mRNA expression levels of MCP-1, IL-6 and VCAM-1 were detected by RT-qPCR, performed using a SYBR PrimeScript RT-PCR kit (Takara Bio, Inc., Otsu, Japan) and the ABI PRISM 7900HT sequence detection system (Applied Biosystems Life Technologies, Foster City, CA, USA). The relative gene expression levels of the target genes, normalized to the endogenous control gene $\beta$-actin, were calculated using the comparative $\mathrm{Ct}$ method formula $2^{-\Delta \Delta \mathrm{CT}}$. The following PCR primers (GenScript Inc, Piscataway, NJ, USA) were used: MCP-1, forward 5'-TGTCCCAAAGAAGCTGTAGT-3' and reverse 5'-ACAGAAGTGCTTGAGGTGGT-3'; IL-6, forward 5'-GTTGCCTTCTTGGGACTGATG-3' and reverse 5'-GTATAGACAGGTCTGTTGGGAG-3'; VCAM-1, forward 5'-TGGCTCCAGACATTTACCCAGTTT-3' and reverse 5'-GTTCTTTGACAGTCTCCCTTTCTTT-3' and $\beta$-actin, forward 5'-CACGATGGAGGGGCCGGACTCATC-3' and reverse 5'-TAAAGACCTCTATGCCAACACAGT-3'. The PCR cycling conditions were as follows: 1 cycle of $50^{\circ} \mathrm{C}$ for $2 \mathrm{~min}, 95^{\circ} \mathrm{C}$ for $10 \mathrm{~min}$, followed by 40 cycles of $95^{\circ} \mathrm{C}$ for $30 \mathrm{sec}$ and $60^{\circ} \mathrm{C}$ for $30 \mathrm{sec}$.

Western blot analysis of the protein levels of MCP-1, IL-6 and $V C A M-1$. The expression levels of proteins in the kidneys were determined using western blot analysis. The tissues, which were frozen in liquid nitrogen following isolation and stored at $-80^{\circ} \mathrm{C}$, were homogenized in $25 \mathrm{mM}$ Tris- $\mathrm{HCl}$ ( $\mathrm{pH}$ 7.4), $1 \mathrm{mM}$ dithiolthreitol and $1 \mathrm{mM}$ sodium orthovanadate (Invitrogen Life Technologies), a protease inhibitor cocktail tablet (complete protease; Roche NimbleGen, Inc., Madison, WI, USA), phosphatase inhibitor cocktail (PhosSTOP; Roche NimbleGen, Inc.) and 1\% Triton X-100 (Invitrogen Life Technologies) using a glass homogenizer (Wuhan Boster Biological Technology, Ltd.). The total protein samples were prepared from the homogenates of pooled arteries and protein concentrations were determined using a bicinchoninic acid protein assay kit (Thermo Fisher Scientific, Waltham, MA, USA). Equal quantities of the protein extracts were separated on 7.5, 10 and $15 \%$ SDS-PAGE gels and immobilized onto polyvinylidene difluoride membranes (EMD Millipore, Billerica, MA, USA). The membranes were then sequentially blocked using Tris-buffered saline containing 5\% non-fat milk and incubated overnight at $4^{\circ} \mathrm{C}$ with the following primary antibodies: Rabbit polyclonal anti-MCP-1 (1:200; ab9669; Abcam, Cambridge, MA, USA); rabbit polyclonal immunoglobulin G (IgG) anti-IL-6 (1:800; sc-1265-R; Santa Cruz Biotechnology, Inc., Dallas, TX, USA) and rabbit polyclonal anti-VCAM-1 (1:600; BS6005; Bioworld Technology, Inc., St. Louis Park, MN, USA). Following incubation, the membranes were washed with Tris-buffered saline (Wuhan Boster Biological Technology, Ltd.) and further incubated with horseradish peroxidase-conjugated goat anti-rabbit polyclonal IgG secondary antibody (1:10,000; Wuhan Boster Biological Technology, Ltd.) at $37^{\circ} \mathrm{C}$ for $1 \mathrm{~h}$. Following several additional washes, immune-positive bands were detected by electrochemiluminescence (Beijing Liuyi Instrument Plant, Beijing, China). Immunoreactive signals were visualized using SuperSignal West Dura Extended Duration Substrate (Thermo Fisher Scientific), and detected using a Kodak XRS system (Eastman Kodak, Fair Lawn, NJ, USA).

Statistical analysis. The data are expressed as the mean \pm standard deviation. Comparison of the means was 


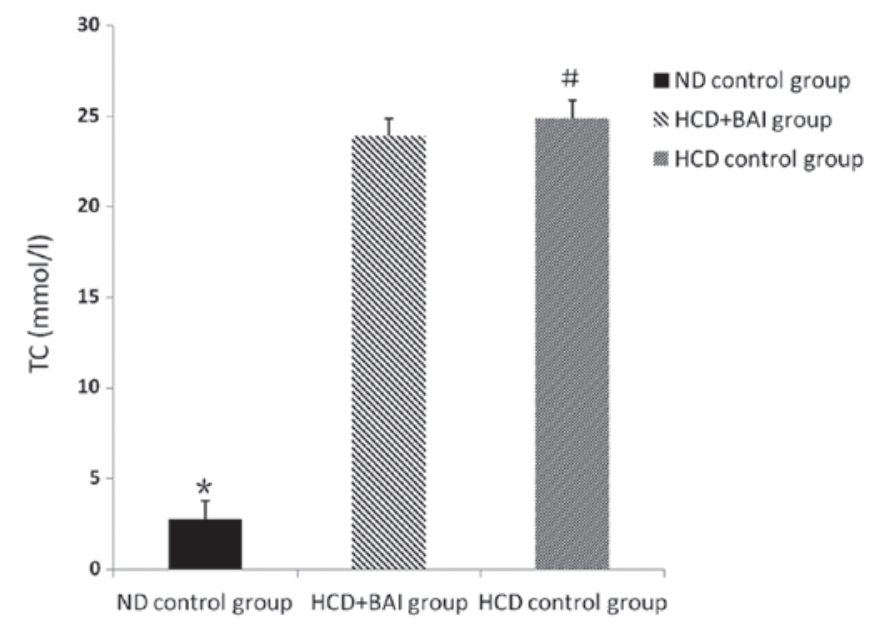

Figure 1. Levels of plasma TC in the mice at 21 weeks. Data are expressed as the mean \pm standard deviation $(n=6) .{ }^{*} \mathrm{P}<0.05$ (ND control, vs. HCD+BAI group); ${ }^{\text {} P}>0.05$ (HCD control, vs. HCD+BAI group). ND; normal diet; HCD, high cholesterol diet; BAI, baicalin; TC, total cholesterol.
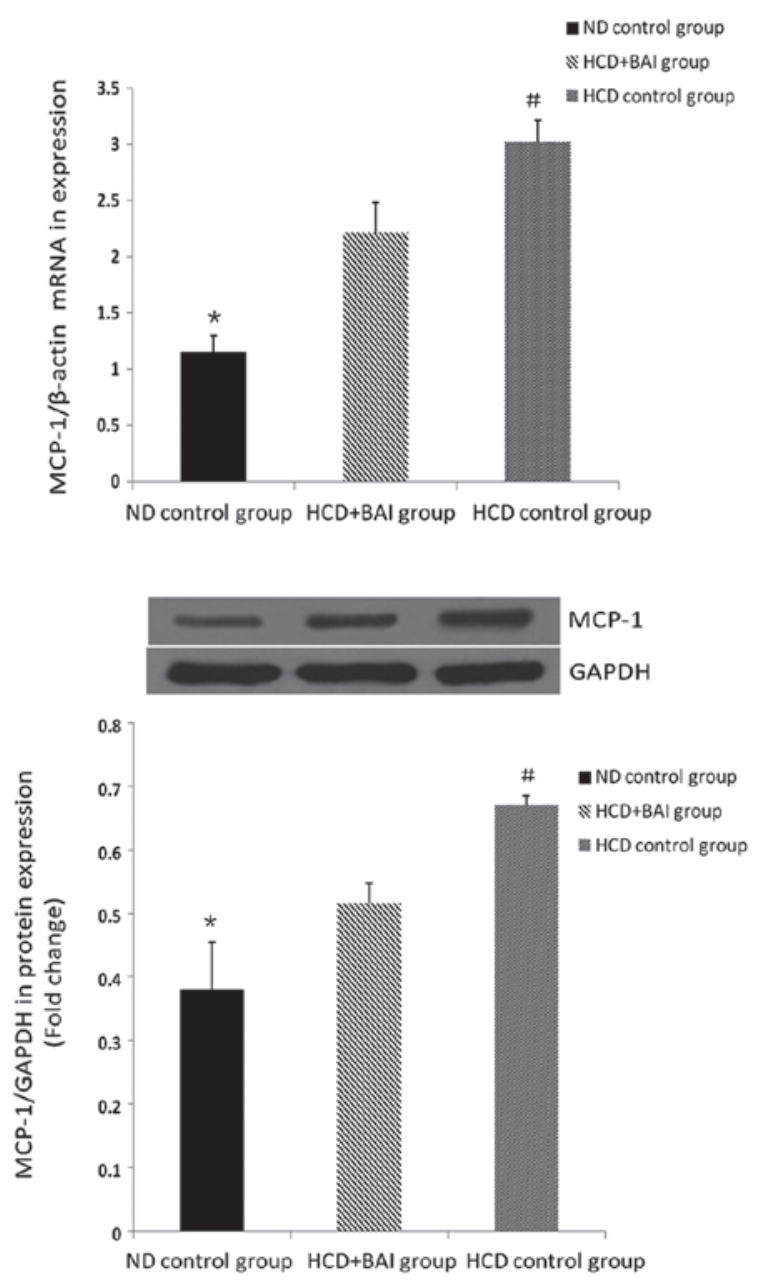

Figure 2. mRNA and protein expression levels of MCP-1 in the kidney tissues of 21-week-old mice. Data are expressed as the mean \pm standard deviation $(\mathrm{n}=6)$. ${ }^{*} \mathrm{P}<0.05$ (ND control, vs., HCD+BAI group); ${ }^{\#} \mathrm{P}<0.05$ (HCD control, vs. HCD+BAI group). MCP-1, monocyte chemoattractant protein-1; ND, normal diet; HCD, high cholesterol diet; BAI, baicalin.
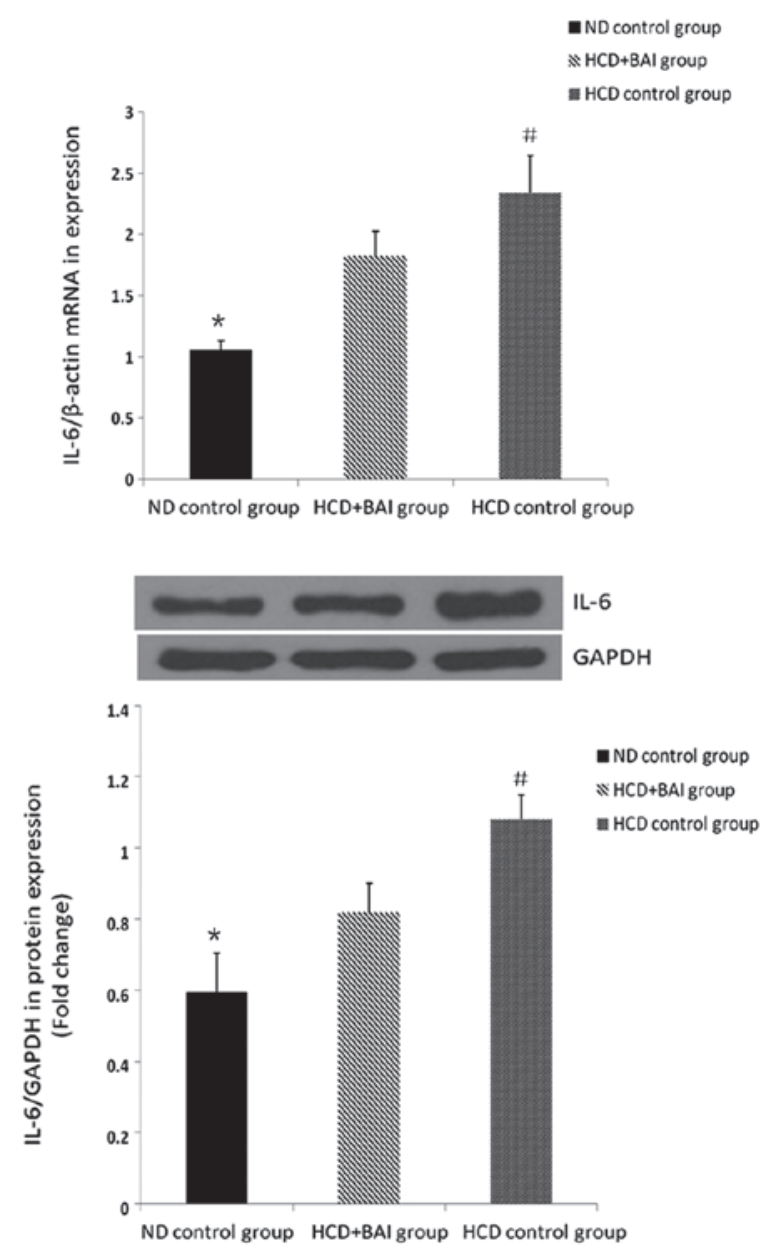

Figure 3. mRNA and protein expression levels of IL-6 in the kidney tissues of 21-week-old-mice. Data are expressed as the mean \pm standard deviation $(\mathrm{n}=6)$. ${ }^{*} \mathrm{P}<0.05$ (ND control, vs. HCD+BAI group); $\mathrm{P}<0.05$ (HCD control, vs. HCD+BAI group). IL-6, interleukin 6; ND, normal diet; HCD, high cholesterol diet; BAI, baicalin.

performed by Student's t-test for two groups and one-way analysis of variance for multiple groups using SPSS 17.0 software (SPSS Inc., Chicago, IL, USA). $\mathrm{P}<0.05$ was considered to indicate a statistically significant difference.

\section{Results}

Impact of baicalin on plasma TC levels in mice. Following the administration of baicalin for 12 weeks, the levels of TC in the male wild-type C57BL/6J mice were lower compared with the ApoE-/- mice fed a high cholesterol diet $(\mathrm{P}<0.05)$. No significant difference in the levels of TC were observed between the baicalin administration group and hypercholesterol control group $(\mathrm{P}>0.05)$. Baicalin caused no decrease in the level plasma TC in the ApoE-/- mice fed a high cholesterol diet (Fig. 1).

$R T-q P C R$ and western blot analysis of the effect of baicalin on the MRNA and protein expression of MCP-1 in the mouse kidneys. To investigate the effect of baicalin on the 

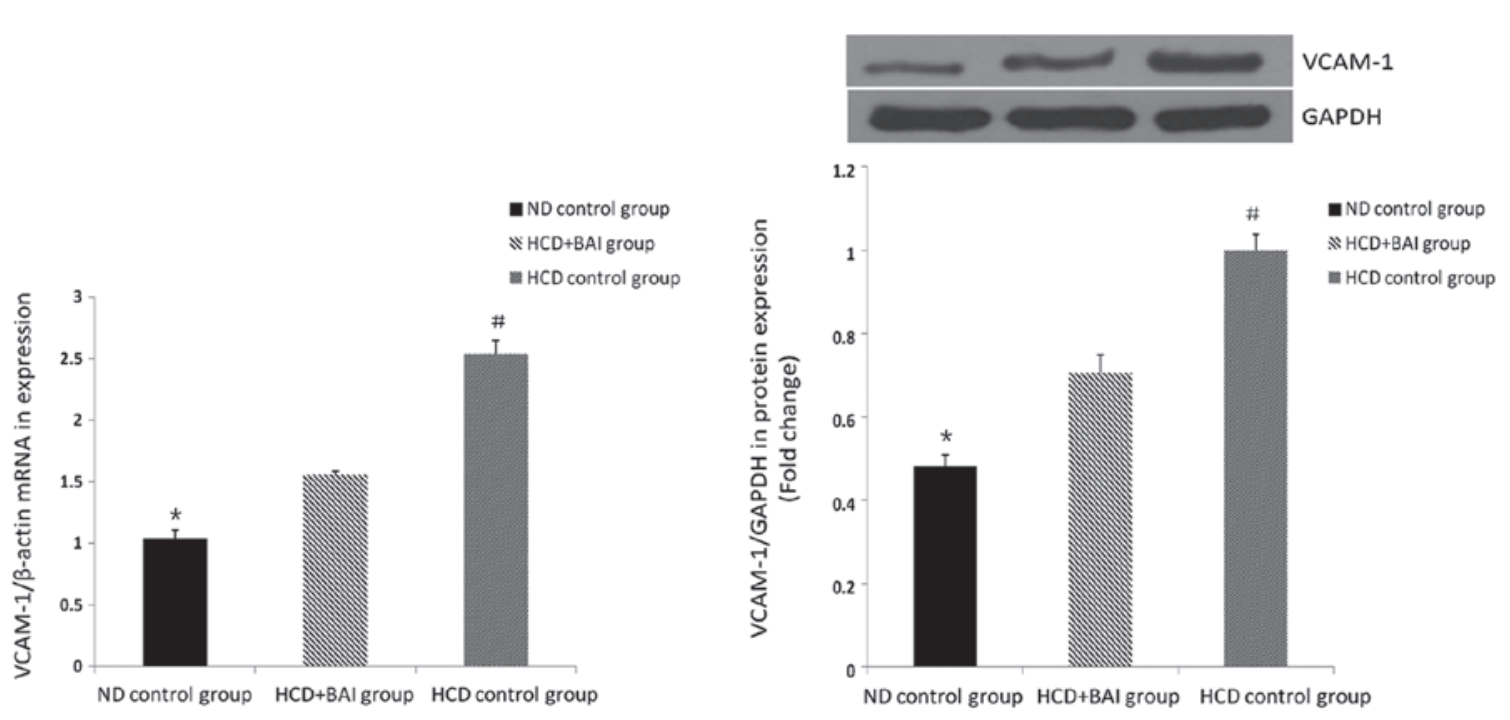

Figure 4. mRNA and protein expression levels of VCAM-1 in the kidney tissues of 21-week-old mice. Data are expressed as the mean \pm standard deviation ( $\mathrm{n}=6) .{ }^{*} \mathrm{P}<0.05$ (ND control, vs. HCD+BAI group); ${ }^{\#} \mathrm{P}<0.05$ (HCD control, vs. HCD+BAI group). VCAM-1, vascular cell adhesion molecule-1; ND, normal diet; HCD, high cholesterol diet; BAI, baicalin.

expression of MCP-1, the mRNA and protein expression levels of MCP-1 were examined in the mouse kidneys. The expression of MCP-1 in the wild-type male C57BL/6J mice was lower compared with all the groups of ApoE-/- mice fed a high cholesterol diet $(\mathrm{P}<0.05)$ and the expression of MCP-1 was highest in the hypercholesterol control group $(\mathrm{P}<0.05)$. Following administration of baicalin, the expression of MCP-1 was reduced compared with that in the hypercholesterol control group $(\mathrm{P}<0.05)$. Baicalin reduced the expression of renal MCP-1 in the ApoE-/- mice fed a high cholesterol $\operatorname{diet}$ (Fig. 2).

RT-qPCR and western blot analysis of the effect of baicalin on the MRNA and protein expression of IL- 6 in the mouse kidneys. To investigate the effect of baicalin on the expression of IL-6, the mRNA and protein expression levels of IL-6 were examined in the mouse kidneys. The expression of IL-6 in the wild-type male C57BL/6J mice was lower compared with all the groups of ApoE-/- mice fed a high cholesterol diet $(\mathrm{P}<0.05)$ and the expression of IL-6 was highest in the hypercholesterol control group $(\mathrm{P}<0.05)$. Following administration of baicalin, the expression of IL- 6 was reduced compared with that in the hypercholesterol control group $(\mathrm{P}<0.05)$. Baicalin also reduced the expression of renal IL- 6 in the ApoE-/- mice fed a high cholesterol diet (Fig. 3).

RT-qPCR and western blot analysis of the effect of baicalin on the $M R N A$ and protein expression of VCAM-1 in the mouse kidneys. The mRNA and protein expression of VCAM-1 was also assessed in the mouse kidneys administered with baicalin. The expression of VCAM-1 in wild-type male C57BL/6J mice was lower compared with all the groups of ApoE-/- mice fed a high cholesterol diet $(\mathrm{P}<0.05)$ and the expression of VCAM-1 was highest in the hypercholesterol control group $(\mathrm{P}<0.05)$. Following baicalin administration, the expression of VCAM-1 was reduced compared with that in the hypercholesterol control group $(\mathrm{P}<0.05)$. Baicalin reduced the expression of renal VCAM-1 in the ApoE-/- mice fed a high cholesterol diet (Fig. 4).

\section{Discussion}

The present study demonstrated that baicalin attenuated the expression levels of MCP-1 and IL-6 in the renal tissues, resulting in suppression of the secretion of VCAM-1 in the ApoE-/- mice fed a high cholesterol diet. However, no decrease in the plasma TC was observed.

Baicalin inhibits the activation of nuclear factor (NF)- $\mathrm{kB}$ via the NF- $\kappa B$-inducing kinase/inhibitor of NF- $\kappa \mathrm{B}$ kinase, extracellular-signal-regulated kinases and p38-mitogen activated protein kinase pathways in the kidney tissues of rats (23). It also significantly suppresses iron overload-induced kidney injury (24) and protects the kidneys of rats with severe acute pancreatitis by inhibiting inflammatory mediators and inducing apoptosis (25). The present study revealed that baicalin attenuated the expression of MCP-1, IL-6 and VCAM-1 in the kidney tissues from the ApoE-/- mice, consistent with previous studies on the inhibition of inflammatory mediators.

As an activator of macrophages and monocytes, MCP-1 recruits macrophages and monocytes, inducing the infiltration of inflammatory cells into the kidney tissues in several types of kidney disease, including diabetic nephropathy (26), glomerulonephritis (27) and chronic renal injury in obesity (28). In the present study, the ApoE-/- mice group fed a high cholesterol diet, exhibited increased expression levels of MCP-1 and IL-6 and this may promote the progression of kidney dysfunction. The results demonstrated that baicalin reduced the expression levels of MCP-1 and IL-6 in the kidney tissues of the AopE-/mice fed a high cholesterol diet. Although no decrease in the level of TC was observed in the mice treated with baicalin, it may inhibit hypercholesterol-associated pro-inflammatory mediators in kidney tissues, reduce leucocyte recruitment into the kidney and alleviate inflammation.

Previous studies have demonstrated that the expression of VCAM-1 increases within the kidneys in several types of kidney diseases, including lupus nephritis (29), chronic renal failure $(30,31)$ and immunoglobulin (Ig)A nephropathy (32). Furthermore, in IgA nephropathy, levels of plasma VCAM-1 
can be used in patients as a marker, not only for severe clinical statuses, but also for severe histological lesions $(32,33)$. Severe hyperlipidaemia in ApoE null mice fed with a $0.15 \%$ cholesterol Western diet leads to glomerular injury, glomerular endothelial cell activation and increased expression levels of major histocompatability complex class II (I-Ab) and VCAM-1, which recruit leucocytes to the kidney and induces renal inflammatory diseases (34). Following treatment with baicalin in the present study, the expression of VCAM-1 in the kidney tissues decreased significantly, accompanied by a decreased production of MCP-1 and IL-6. Therefore, the inhibitory effect of baicalin may be due to its ability to attenuate pro-inflammatory producers under hyperlipidemia.

The present study had certain limitations. Baicalin was not compared with other drugs and only a single concentration of baicalin, in accordance with previous studies, was used. Therefore, no comparison was made on the effects of different concentrations of baicalin.

In conclusion, the present study demonstrated that the administration of baicalin induced a substantial decrease in the expression levels of MCP-1, IL-6 and VCAM-1 in the kidney tissue from ApoE-/- mice fed a high cholesterol diet and revealed that baicalin may prevent the process of inflammatory mediator-induced kidney dysfunction in several types of renal disease.

\section{References}

1. Moorhead JF, Chan MK, El-Nahas M and Varghese Z: Lipid nephrotoxicity in chronic progressive glomerular and tubulo-interstitial disease. Lancet 2: 1309-1311, 1982.

2. uan XZ, Varghese Z and Moorhead JF: An update on the lipid nephrotoxicity hypothesis. Nat Rev Nephrol 5: 713-721, 2009.

3. Kim E, Tolhurst AT, Qin LY, Chen XY, Febbraio M and Cho S: CD36/fatty acid translocase, an inflammatory mediator, is involved in hyperlipidemia-induced exacerbation in ischemic brain injury. J Neurosci 28: 4661-4670, 2008.

4. Sultan A, Strodthoff D, Robertson AK, et al: T cell-mediated inflammation in adipose tissue does not cause insulin resistance in hyperlipidemic mice. Circ Res 104: 961-968, 2009.

5. Perez de Lema G, Maier H, Nieto E, et al: Chemokine expression precedes inflammatory cell infiltration and chemokine receptor and cytokine expression during the initiation of murine lupus nephritis. J Am Soc Nephrol 12: 1369-1382, 2001.

6. Anders HJ, Belemezova E, Eis V, et al: Late onset of treatment with a chemokine receptor CCR1 antagonist prevents progression of lupus nephritis in MRL-Fas(lpr) mice. J Am Soc Nephrol 15: 1504-1513, 2004.

7. Rovin BH, Rumancik M, Tan L and Dickerson J: Glomerular expression of monocyte chemoattractant protein-1 in experimental and human glomerulonephritis. Lab Invest 71: 536-542, 1994.

8. Zoja C, Liu XH, Donadelli R, et al: Renal expression of monocyte chemoattractant protein-1 in lupus autoimmune mice. J Am Soc Nephrol 8: 720-729, 1997 .

9. Tesch GH, Schwarting A, Kinoshita K, Lan HY, Rollins BJ and Kelley VR: Monocyte chemoattractant protein-1 promotes macrophage-mediated tubular injury, but not glomerular injury, in nephrotoxic serum nephritis. J Clin Invest 103: 73-80, 1999.

10. Chow F, Ozols E, Nikolic-Paterson DJ, Atkins RC and Tesch GH: Macrophages in mouse type 2 diabetic nephropathy: correlation with diabetic state and progressive renal injury. Kidney Int 65: 116-128, 2004.

11. He X, Schoeb TR, Panoskaltsis-Mortari A, et al: Deficiency of P-selectin or P-selectin glycoprotein ligand-1 leads to accelerated development of glomerulonephritis and increased expression of $\mathrm{CC}$ chemokine ligand 2 in lupus-prone mice. J Immunol 177: 8748-8756, 2006.

12. Tashiro K, Koyanagi I, Saitoh A, et al: Urinary levels of monocyte chemoattractant protein-1 (MCP-1) and interleukin-8 (IL-8), and renal injuries in patients with type 2 diabetic nephropathy. J Clin Lab Anal 16: 1-4, 2002.
13. Kanamori $\mathrm{H}$, Matsubara $\mathrm{T}$, Mima $\mathrm{A}$, et al: Inhibition of MCP-1/CCR2 pathway ameliorates the development of diabetic nephropathy. Biochem Biophys Res Commun 360: 772-777, 2007.

14. Chow FY, Nikolic-Paterson DJ, Ozols E, Atkins RC, Rollin BJ and Tesch GH: Monocyte chemoattractant protein-1 promotes the development of diabetic renal injury in streptozotocin-treated mice. Kidney Int 69: 73-80, 2006.

15. Garibotto G, Sofia A, Balbi M, et al: Kidney and splanchnic handling of interleukin-6 in humans. Cytokine 37: 51-54, 2007.

16. Li C, Lin G and Zuo Z: Pharmacological effects and pharmacokinetics properties of Radix scutellariae and its bioactive flavones. Biopharm Drug Dispos 32: 427-445, 2011.

17. Baylor NW, Fu T, Yan YD and Ruscetti FW: Inhibition of human $\mathrm{T}$ cell leukemia virus by the plant flavonoid baicalin (7-glucuronic acid, 5,6-dihydroxyflavone). J Infect Dis 165: 433-437, 1992.

18. Li BQ, Fu T, Dongyan Y, Mikovits JA, Ruscetti FW and Wang JM: Flavonoid baicalin inhibits HIV-1 infection at the level of viral entry. Biochem Biophys Res Commun 276: 534-538, 2000.

19. Zeng Y, Song C, Ding X, Ji X, Yi L and Zhu K: Baicalin reduces the severity of experimental autoimmune encephalomyelitis. Braz J Med Biol Res 40: 1003-1010, 2007.

20. Zhang XP, Tian H, Wu DJ, et al: Pathological changes in multiple organs of rats with severe acute pancreatitis treated by baicalin and octreotide. Hepatobiliary Pancreat Dis Int 8: 85-92, 2009.

21. Zhang X, Tian H, Wu C, et al: Effect of baicalin on inflammatory mediator levels and microcirculation disturbance in rats with severe acute pancreatitis. Pancreas 38: 732-738, 2009.

22. Zwai M, Chen R, Li Z, et al: Deletion of angiotensin II type 2 receptor exaggerated atherosclerosis in apolipoprotein E-null mice. Circulation 112: 1636-1643, 2005.

23. Kim DH, Kim HK, Park S, et al: Short-term feeding of baicalin inhibits age-associated NF-kappaB activation. Mech Ageing Dev 127: 719-725, 2006.

24. Zhang Y, Gao Z, Liu J and Xu Z: Protective effects of baicalin and quercetin on an iron-overloaded mouse: comparison of liver, kidney and heart tissues. Nat Prod Res 25: 1150-1160, 2011 .

25. Zhang XP, Tian H, Lai YH, et al: Protective effects and mechanisms of Baicalin and octreotide on renal injury of rats with severe acute pancreatitis. World J Gastroenterol 13: 5079-5089, 2007.

26. Lv SS, Liu G, Wang JP, et al: Mesenchymal stem cells transplantation ameliorates glomerular injury in streptozotocin-induced diabetic nephropathy in rats via inhibiting macrophage infiltration. Int Immunopharmacol 17: 275-282, 2013.

27. Tesch GH, Maifert S, Schwarting A, Rollins BJ and Kelley VR: Monocyte chemoattractant protein 1-dependent leukocytic infiltrates are responsible for autoimmune disease in MRL-Fas(lpr) mice. J Exp Med 190: 1813-1824, 1999.

28. Fu CP, Lee IT, Sheu WH, et al: The levels of circulating and urinary monocyte chemoattractant protein-1 are associated with chronic renal injury in obese men. Clin Chim Acta 413: 1647-1651, 2012.

29. Wu T, Xie C, Wang HW, et al: Elevated urinary VCAM-1, P-selectin, soluble TNF receptor-1, and CXC chemokine ligand 16 in multiple murine lupus strains and human lupus nephritis. J Immunol 179: 7166-7175, 2007.

30. Bolton CH, Downs LG, Victory JG, et al: Endothelial dysfunction in chronic renal failure: roles of lipoprotein oxidation and pro-inflammatory cytokines. Nephrol Dial Transplant 16: 1189-1197, 2001.

31. Cottone S, Mule G, Amato F, et al: Amplified biochemical activation of endothelial function in hypertension associated with moderate to severe renal failure. J Nephrol 15: 643-648, 2002.

32. Zhu L, Shi S, Liu L, Lv J and Zhang H: Increased plasma sVCAM-1 is associated with severity in IgA nephropathy. BMC Nephrol 14: 21, 2013.

33. Nelson CL, Karschimkus CS, Dragicevic G, et al: Systemic and vascular inflammation is elevated in early IgA and type 1 diabetic nephropathies and relates to vascular disease risk factors and renal function. Nephrol Dial Transplant 20: 2420-2426, 2005.

34. Bruneval P, Bariety J, Belair MF, Mandet C, Heudes D and Nicoletti A: Mesangial expansion associated with glomerular endothelial cell activation and macrophage recruitment is developing in hyperlipidaemic apoE null mice. Nephrol Dial Transplant 17: 2099-2107, 2002. 